\title{
Projeto e Desenvolvimento de um Sistema de Mo- nitoramento dos Níveis de Pressão Sonora
}

\author{
Project and Development of a Sound Pressure Level Monitoring
}

Gabriel Cysneiros Bezerra Carvalho Oliveira

Escola Politécnica de Pernambuco

Universidade de Pernambuco

50.720-001 - Recife, Brasil

gabrielcbco@live.co

Resumo O presente artigo descreve a construção de um sistema de monitoramento do nível de pressão sonora em ambiente hospitalar utilizando a plataforma do Arduino que emite um sinal luminoso quando o nivel recomendado pela NBR 10.152 é ultrapassado. Os dados obtidos são armazenados em um cartão $S D$ afim de que sejam estudados e encontradas soluções para reduzir barulhos em hospital. Apresenta os resultados dos primeiros testes realizados.

Palavras-Chave: Pressão Sonora, Ambiente Hospitalar, Arduino.Decibelímetro

\footnotetext{
Abstract This article describes the assemblying of a sound level pressure monitoring system in a hospital room using the Arduino platfor that sends a light signal whem the recommended limit on NBR 10.152 is exceeded. Data is stored on a SD card to further studies to find solutions to reduce noise in hospital rooms. First results are presented.
}

Keywords: Sound Pressure Level, Hospital Room, Arduino.Decibelmeter 


\section{Introdução}

A poluição sonora ambiental não é um problema recente e hoje em dia torna-se onipresente, chegando a beirar o intolerável. Quase não há locais livre do excesso de ruído, podendo expor-se a ele dentro de casa, na rua, no trabalho e em vários outros locais.

Um local que parecia estar livre da ocorrência deste tipo de poluição seria os hospitais, porém, em uma cidade grande, torna-se quase impossivél isola-los de fontes de ruído externo como o transito de grandes avenidas, carros de som, etc. Entretanto, muito do ruído hospitalar provém de dentro do proprío hospital, gerados pelas conversações da equipe hospitalar e também pelos equipamentos dotados de alarmes sonoros essenciais para alertar médicos e enfermeiros sobre as mudanças nas condições clinicas de seus pacientes, ou mau funcionamento do equipamento. Assim, um ambiente que deveria ser sinônimo de descanso torna-se um ambiente inapropriado para o mesmo, como mostra estudo conduzido em hospital no Paraná [1].

Além do mais, a exposição ao ruído intenso está associada à várias manifestações sistêmicas tais como aumentar a sensibilidade do paciente à dor, aceleração da frequência cardíaca e respiratória e dificutar a sedação do paciente, pois o mesmo sentirá mais dificuldade para dormir[2]. Por isso, a Associação Brasileira de Normas Técnicas (ABNT) define, na NBR 10.512, que o ruído dentro de apartamentos, bercários, centro cirúrgicos e enfermarias deve estar entre 35 e $45 \mathrm{~dB}$ [3].

\section{Objetivo}

Desenvolver um sistema de monitoramento dos níveis de pressão sonora em ambientes hospitalares, armazenando os dados em um cartão de memória e emitindo um sinal luminoso quando o limite aceitável for ultrapassado , detectar os motivos pelos quais esse limite é ultrapassado e estudar maneiras de soluciona-los.

\section{Método}

O sistema capta o ruído através de um microfone tipo dinâmico com padrão polar supercardióide. Esse gera uma resposta muito baixa[4], que precisa ser amplificada e passar por uma filtragem ponderada do tipo A[5], que enfatiza as frequências de $1 \mathrm{kHz}$ a $5 \mathrm{kHz}$ (onde o ouvido humano é mais sensível), conforme a Figura 1[6]. A seguir, o sinal entrará no Arduino é retificado[7], pois o mesmo não suporta tensões negativas. Um programa irá transformar o sinal recebido em decibéis seguindo o fluxograma mostrado Figura 2. A fórmula para transformar o sinal de entrada em decibel é mostrada na equação (1). Porém, como o Arduino não possui uma biblioteca que nos auxilie na resolução de integral, a mesma é calculada utilizando a equação (2).

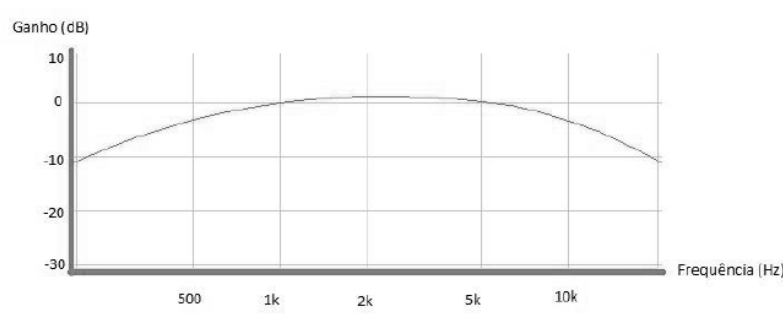

Figura 1- Resposta em frequência para o filtro ponderado tipo $A$

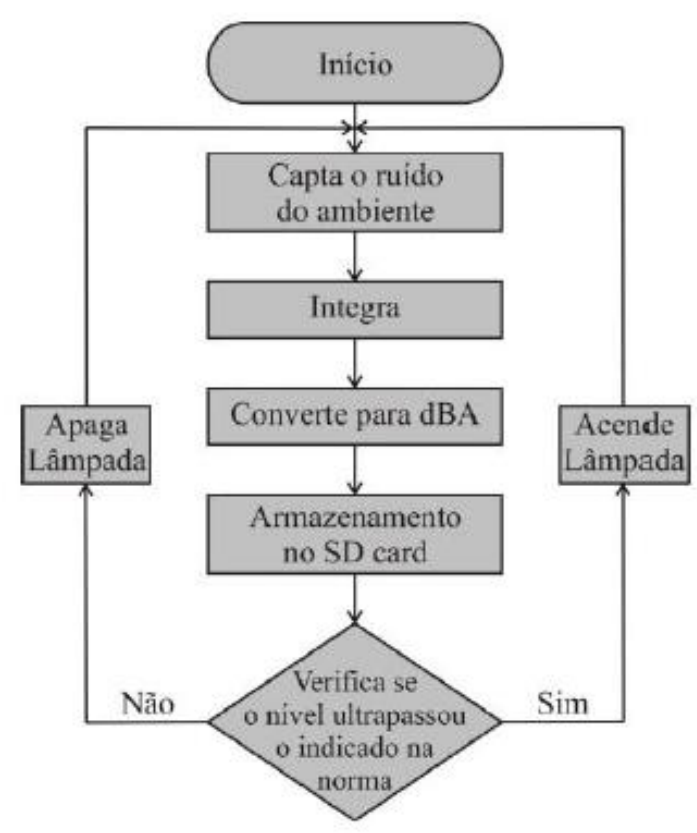

Figura 2 - Fluxograma do código no Arduino

$N(d B)=10 \log \left(\frac{\int_{0}^{T} \operatorname{Vin}^{2}(t) d t}{\int_{0}^{T} \operatorname{Vref}^{2}(t) d t}\right)$

$\int_{0}^{T} \operatorname{Vin}^{2}(t) d t=\Delta T \sum_{i=1}^{N} \frac{\operatorname{Vin}_{\left(t_{i-1}\right)}^{2}+\operatorname{Vin}_{\left(t_{i}\right)}^{2}}{2}$

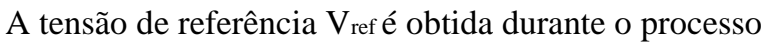
de calibração. Nesse processo, o valor em decibel obtido no Arduino é comparado com os valores em um decibelimetro digital HDR 822, fabricado pela Hikari (Figura 
3) e outro decibelimetro DL 4200, fabricado pela Icel (Figura 4), ambos com calibração de fábrica. Então, fazendo uma manipulação matemática na equação (1), temos que:

$$
10 \log V_{r e f}=N(d B)-10 \log V_{i n}=C
$$

Onde, na equação (3), N(dB) é o valor obtido pelo decibelimetro e $10 \log \mathrm{V}_{\text {in }}$ é obtido pelo valor do Arduino. Dessa forma, o termo à direita da equação (3) é uma constante conhecida e pode ser chamada de C. Assim, o valor

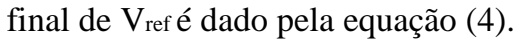

$$
V_{r e f}=10^{c / 10}
$$

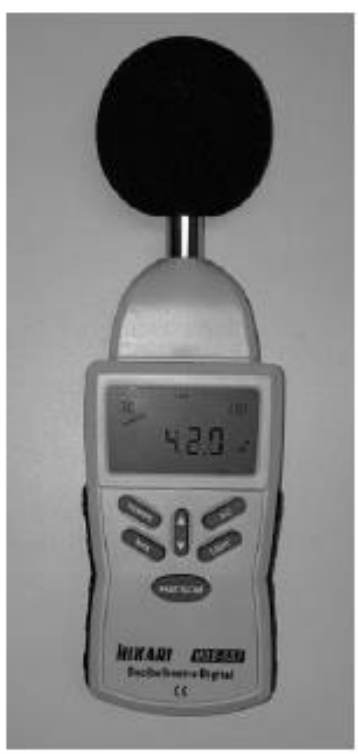

Figura 3 - Decibelimetro HDR 822, fabricado pela Hikari

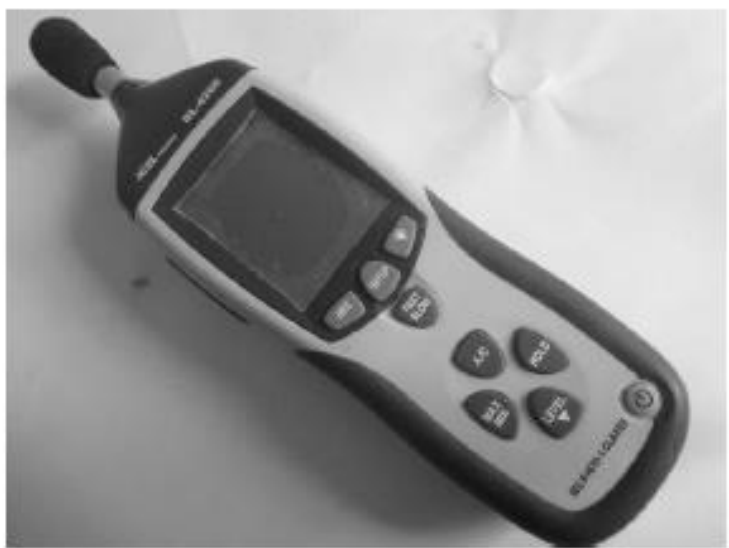

Figura 4 - Decibelimetro DL 4200, fabricado pela Icel
Com o sistema calibrado, é necessário então associar o valor obtido com o horário em que ele foi coletado. Para isso, utilizou-se um módulo RTC (Real Time Clock da Figura 5) que usa um programa no Arduino para retornar a hora, minuto, segundo, dia, mês e ano no qual o dado foi coletado[8], criando-se então um datalogger. As ligações necessárias entre o módulo RTC e o Arduino são mostradas na Tabela 1.

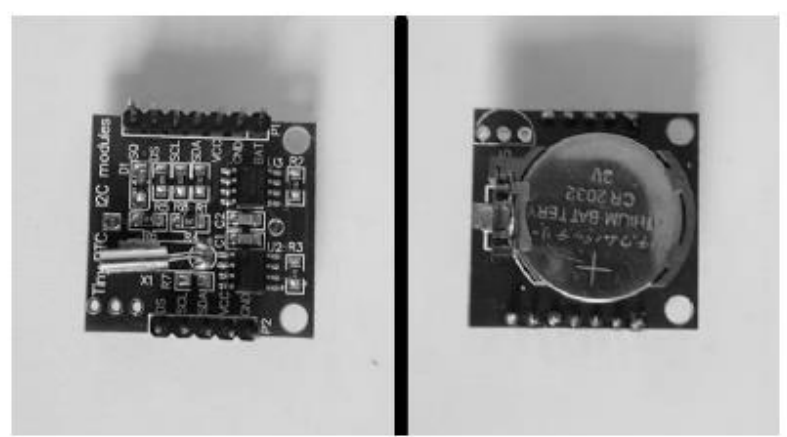

Figura 5 - Vista superior e inferior do módulo RTC

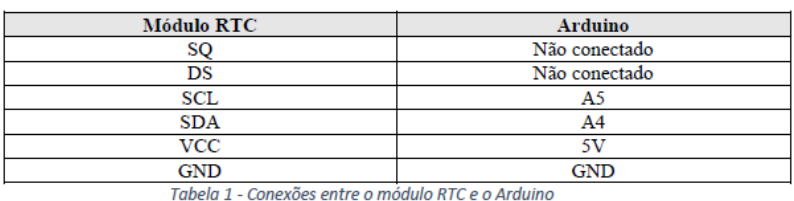

Para armazenar os resultados num cartão de memória SD, utilizou-se um módulo SD Shield fabricado pela LC Studio e um cartão SD de 4 GB (Figura 6). As conexões entre o SD Shield e o Arduino são mostradas na Tabela 2. Para que o módulo funcionasse corretamente, é necessário adicionar a biblioteca SD no Arduino[9].

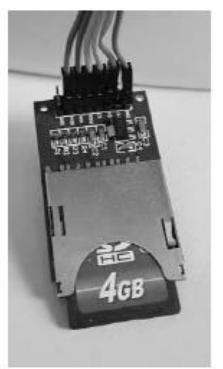

Figura 6 - Módulo SD Shield fabricado pela LC Studio e cartão SD de 4GB

\begin{tabular}{|c|c|}
\hline Módulo SD Shield & Ardino \\
\hline 3.3V & Não conectado \\
\hline GND & GND \\
\hline CS & 4 \\
\hline MOSI & 11 \\
\hline SCK & 13 \\
\hline MISO & 12 \\
\hline Tabela 2 - Conexões entre o módulo SD Shield e o Arduino \\
\hline
\end{tabular}


Quando o limite sonoro definido pela NBR 10.152 é ultrapassado, uma lâmpada é acesa. Análises serão realizadas para avaliar o comportamento dos profissionais de saúde, no seu ambiente de trabalho, em dois momentos: Sem saber da monitoração e já informados de que estão sendo monitorados. $\mathrm{O}$ sistema completo é mostrado na Figura 7.

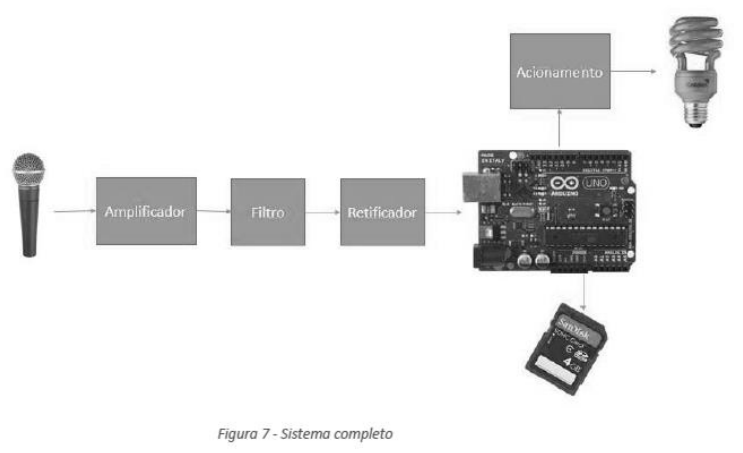

\section{Resultados}

O sistema foi montando utilizando um Software de Simulação e uma placa de circuito impresso foi gerada utilizando o layout da Figura 8. Após soldar os componentes eletrônicos, o sistema ficou como mostrado na Figura 9.

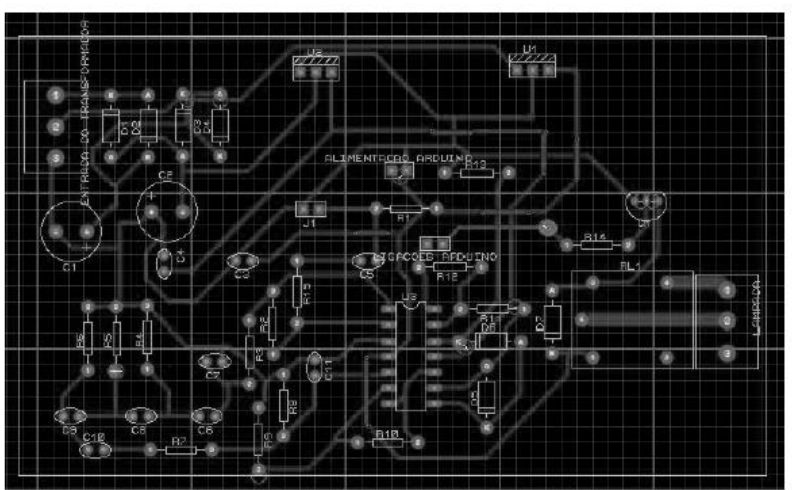

Figura 8 - Design da placa de circuito impresso montada para o sistema

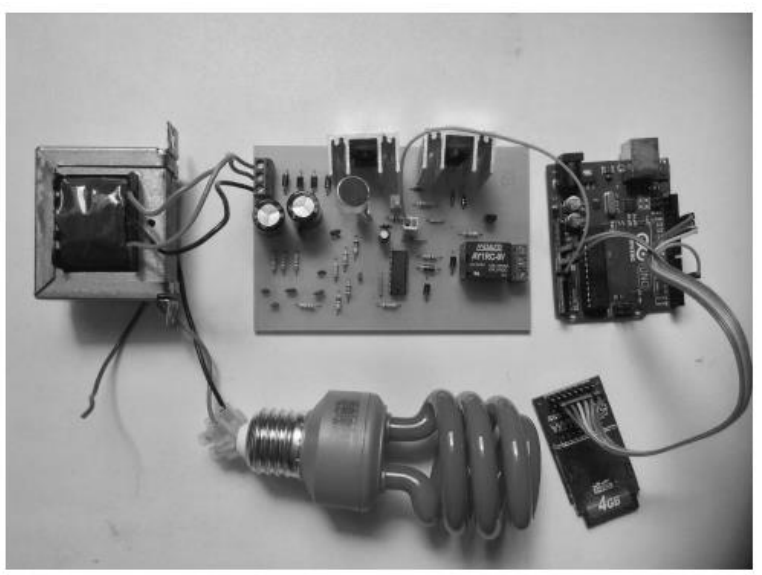

Figura 9 - Sistema completo montado em bancada

Um primeiro teste foi realizado e os valores obtidos foram comparados com os decibelímetros disponíveis e o resultado é mostrado no Gráfico 1. Calculando a tensão de referência de acordo com a equação (4), obtemos o resultado $\mathrm{V}_{\text {ref }}=0,000000763856304$. Após a utilização da nova tensão de referência, o resultado obtido é mostrado no Gráfico 2.

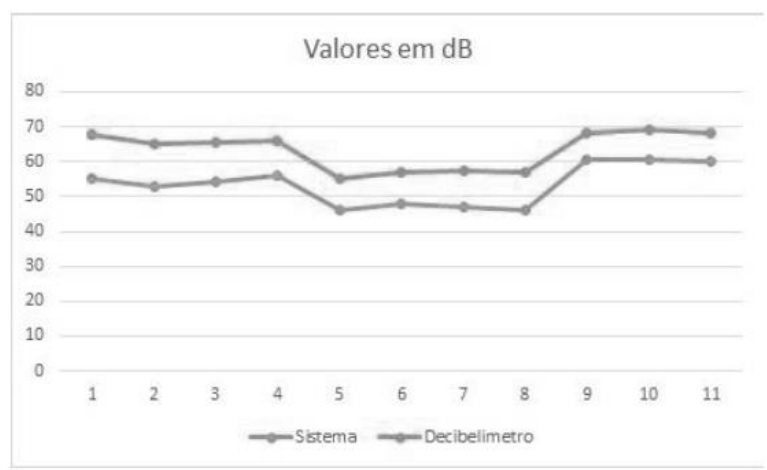

Gráfico 1 - Valores obtidos no primeiro teste e comparado com um decibelimetro

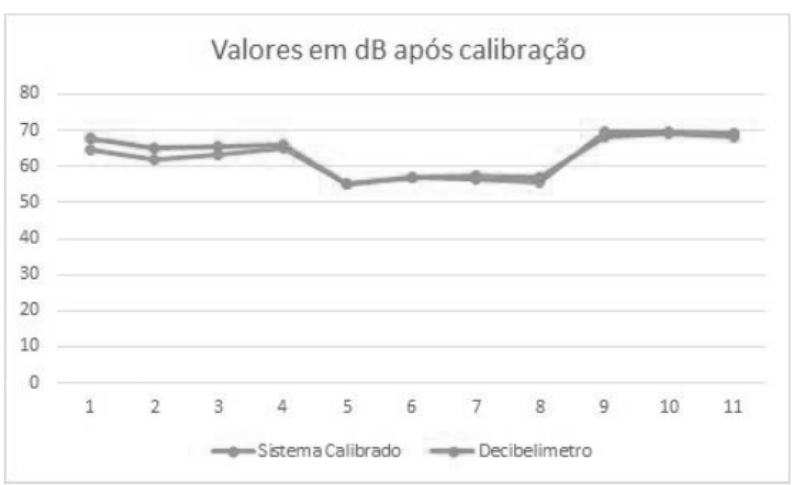

Gráfico 2 - Resultados obtido após a calibração e comparados com um decibelimetro

Após a utilização do módulo RTC para criar o datalogger, foi possível armazenar os dados no cartão SD no formato de arquivo .txt. Uma parte dos resultados coletados em um teste residencial é mostrado na Figura 10. Aqui 
é possível ver o momento exato no qual o dado foi coletado.

$$
\begin{aligned}
& \text { 22:25:05 13/12/15 Valor em dB: } 73.35 \\
& \text { 22:25:07 13/12/15 Valor em dB: } 72.36 \\
& \text { 22:25:09 13/12/15 Valor em dB: } 72.42 \\
& \text { 22:25:11 13/12/15 Valor em dB: } 72.41 \\
& \text { 22:25:14 13/12/15 Valor em dB: } 74.03 \\
& \text { 22:25:16 13/12/15 Valor em } d B: 72.94 \\
& \text { 22:25:18 13/12/15 Valor em dB: } 73.39 \\
& \text { 22:25: } 20 \text { 13/12/15 Valor em dB: } 72.99 \\
& \text { 22: } 25: 22 \text { 13/12/15 Valor em dB: } 72.69 \\
& 22: 25: 24 \text { 13/12/15 Valor em dB: } 64.37 \\
& \text { 22:25:26 13/12/15 Valor em dB: } 64.31 \\
& \text { 22:25:29 13/12/15 Valor em dB: } 64.27 \\
& \text { 22:25: } 31 \text { 13/12/15 Valor em dB: } 64.23 \\
& \text { 22:25:33 13/12/15 Valor em dB: } 64.19 \\
& \text { 22:25:35 13/12/15 Valor em dB: } 64.16 \\
& \text { 22: } 25: 37 \text { 13/12/15 Valor em dB: } 64.14 \\
& \text { 22:25:39 13/12/15 Valor em dB: } 64.14 \\
& \text { 22:25:42 13/12/15 Valor em dB: } 64.12
\end{aligned}
$$

\section{Figura 10 - Resultados dentro do arquivo .txt}

\section{Conclusão}

Analisando o Gráfico 2 percebe-se que o Sistema apresenta uma resposta similar à resposta dos decibelimetros utilizados para calibração, porém, o sistema ainda precisa de uma melhor calibração, utilizando valores mais elevados de ruído e uma faixa mais ampla de frequências, antes de ser implantado em um ambiente hospitalar. Após essa implantação, espera-se uma mudança comportamental nos profissionais de saúde, reduzindo o barulho dentro dos hospitais e auxiliando na recuperação dos pacientes.

Outra conclusão importante vem dos dados obtidos durante a calibração. Alcançar valores entre 35 e $45 \mathrm{~dB}$, como recomenda a ABNT, é algo muito difícil. Então esse estudo também pode ser usado para sugestões de revisão junto à ABNT.

Vale salientar que todo o sistema foi montado, inicialmente, com um microfone de eletreto para que os primeiros testes pudessem ser realizados. A intensão é utilizar um outro microfone do tipo dinâmico. O microfone escolhido foi o GS 36, fabricado pela Alltech. O mesmo já foi adquirido e é mostrado na Figura 11.

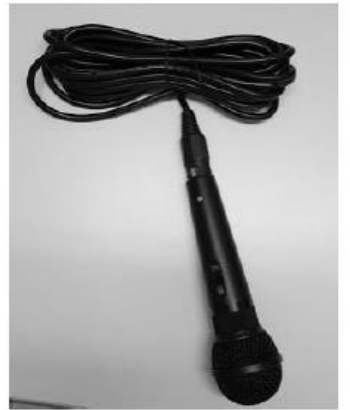

Figura 11 - Microfone GS 36 fabricado pela Alltech. Será utilizado em etapas futuras do projeto.

\section{Referências}

[1] Otenio, M.H. et al. Intensidade de ruído em hospital de 222 leitos da 18 regional de saúde - PR. Brás Otorrinolaringol. 2007.

[2] Posso, M. B. S. O ruído emitido por aparelhos usados nas salas de operacões. 98f. 1980. Dissertação (Mestrado) - Escola de Enfermagem da USP, 1980.

[3] ASSOCIAÇÃO BRASILEIRA DE NORMAS TÉCNICAS (ABNT). NBR 10.152 - Avaliação do ruído para o conforto acústico. Rio de Janeiro: ABNT, 5 p. Dez. 1987.

[4] Platt, Charles. Electronics: Fun \& Fundamentals - The Eclectic Electret Microphone. Disponível em: http://makezine.com/magazine/make-39/electronics-fun-fundamentals-theeclectic-electret-microphone/, Mar. 2015

[5] BEIS, Uwe. Weighting Filter Set. Disponível em:

http://www.beis.de/Elektronik/Audi-

oMeasure/WeightingFilters.html , Mar. 2015

[6] SPADA, A. L. O Ouvido Humano. Disponível em: http://www.attack.com.br/artigos_tecnicos/ouvido_humano.pdf

[7] Ye, Ting. Precision Full-Wave Rectifier, Dual Supply. Texas Instruments. Dez. 2013.

[8] Boxall, John. Tutorial - Using DS1307 and DS3231 Real Time Clock Modules with Arduino. Tronixlabs. Dez. 2014. Disponível em: http://tronixstuff.com/2014/12/01/tutorial-usingds1307-and-ds3231-real-time-clock-moduleswith-arduino/

[9] Arduino. SD Library. Disponível em: https://www.arduino.cc/en/Reference/SD. 